\title{
Surgery for metachronous metastasis of soft tissue sarcoma - A magnitude of benefit analysis using propensity score methods
}

\author{
Maria A. Smolle a, b, *,1, Veroniek M. van Praag c, ${ }^{\text {, Florian Posch }}{ }^{\text {b, d, }}$, Marko Bergovec ${ }^{\text {a, b }}$, \\ Lukas Leitner ${ }^{\mathrm{a}, \mathrm{b}}$, Jörg Friesenbichler ${ }^{\mathrm{a}, \mathrm{b}}$, Ronald Heregger ${ }^{\mathrm{b}, \mathrm{d}}$, Jakob M. Riedl ${ }^{\mathrm{b}, \mathrm{d}}$, \\ Martin Pichler b, d, e, Armin Gerger b, d, Joanna Szkandera ${ }^{\mathrm{b}, \mathrm{d}}$, Herbert Stöger ${ }^{\mathrm{b}, \mathrm{d}}$,' \\ Freyja-Maria Smolle-Jüttner ${ }^{\text {b, f }}$, Bernadette Liegl-Atzwanger ${ }^{\text {b, g, }}$, Marta Fiocco ${ }^{\text {c, h, i }}$, \\ Michiel AJ. van de Sande ${ }^{c}$, Andreas Leithner ${ }^{\mathrm{a}, \mathrm{b}}$

\footnotetext{
a Department of Orthopaedics and Trauma, Medical University of Graz, Auenbruggerplatz 5, 8036, Graz, Austria

b Comprehensive Cancer Centre Graz, Graz, Austria

c Department of Orthopaedic Surgery, Leiden University Medical Centre, Albinusdreef 2, 2333 ZA, Leiden, The Netherlands

d Division of Oncology, Department of Internal Medicine, Medical University of Graz, Auenbruggerplatz 15, 8036, Graz, Austria

e Department of Experimental Therapeutics, The UT MD Anderson Cancer Center, Sout Campus Research Building 4, 1901 East Road, Houston, TX, USA

${ }^{\mathrm{f}}$ Division of Thoracic and Hyperbaric Surgery, Medical University of Graz, Auenbruggerplatz 29, 8036, Austria

${ }^{g}$ Institute of Pathology, Medical University of Graz, Auenbruggerplatz 25, 8036, Graz, Austria

${ }^{\mathrm{h}}$ Department of Medical Statistics and Bioinformatics, Leiden University Medical Centre, Albinusdreef 2, 2333 ZA, Leiden, The Netherlands
} \\ ${ }^{\mathrm{i}}$ Mathematical Institute Leiden University, Albinusdreef 2, 2333 ZA, Leiden, The Netherlands
}

\section{A R T I C L E I N F O}

Article history:

Accepted 20 June 2018

Available online $\mathrm{xxx}$

Keywords:

Soft tissue sarcoma

Metastasis

Overall-survival

Metastasectomy

\begin{abstract}
A B S T R A C T
Introduction: Metastasectomy is hypothesised to improve OS in metastatic STS, but evidence in favour of this approach derives from non-controlled single-arm cohorts affected by selection bias. The objective was to quantify the effect of metastasectomy vs. non-surgical management on overall survival (OS) in patients with metachronous metastases from extremity- and trunk soft tissue sarcoma (STS).

Materials and methods: From a population of 1578 STS patients, 135 patients who underwent surgery for localised STS at two European centres between 1998 and 2015 and developed metachronous STS metastases were included. Propensity score analyses with inverse-probability-of-treatment-weights (IPTW) and landmark analyses were performed to control for selection and immortal time bias, respectively. Results: OS was significantly longer in the 68 patients undergoing metastasectomy than in the 67 patients who were treated non-invasively for their metastasis (10-year OS: $23 \%$ vs. $4 \%$; hazard ratio $(\mathrm{HR})=0.34,95 \% \mathrm{CI}: 0.22-0.53, \mathrm{p}<0.0001$ ). This association prevailed after IPTW-weighting of the data to control for the higher prevalence of favourable prognostic factors in the surgery group (adjusted 10year OS: $17 \%$ vs. $3 \%$, log-rank p $<0.0001$; $\mathrm{HR}=0.33,95 \% \mathrm{CI}: 0.20-0.52, \mathrm{p}<0.0001$ ). Five-year OS estimates were $27.8 \%$ in patients who had and $14.5 \%$ in patients who had not undergone metastasectomy within the first 3 months after diagnosis of a metastasis $(p<0.0001)$.

Conclusion: In this observational bi-centre study, metastasectomy was associated with prolonged survival in patients with metachronous STS metastases. In the absence of randomized studies, our results indicate that metastasectomy should be considered as an important treatment option for metachronous STS metastases.
\end{abstract}

( 2018 Elsevier Ltd, BASO The Association for Cancer Surgery, and the European Society of Surgical Oncology. All rights reserved.

* Corresponding author. Department of Orthopaedics and Trauma, Medical University of Graz, Auenbruggerplatz 5, 8036, Graz, Austria.

E-mail addresses: maria.smolle@medunigraz.at (M.A. Smolle), v.m.van_praag@lumc.nl (V.M. van Praag), florian.posch@medunigraz.at (F. Posch), marko.bergovec@

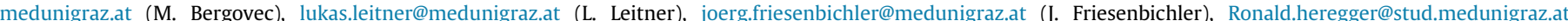

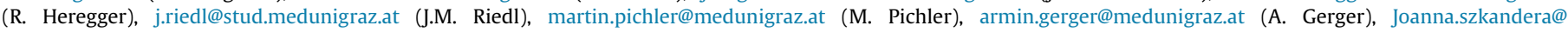

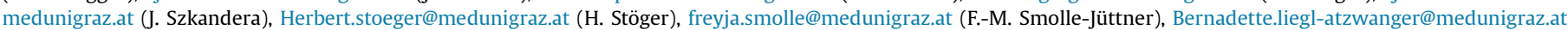
(B. Liegl-Atzwanger), m.fiocco@lumc.nl (M. Fiocco), m.a.j.van_de_Sande@lumc.nl (M.AJ. van de Sande), andreas.leithner@medunigraz.at (A. Leithner).

1 These authors contributed equally. 


\section{Introduction}

Soft tissue sarcomas (STS) constitute a group of rare mesenchymal tumours with diverse molecular, histologic, and clinical features [1]. While a significant number of patients with localised STS can be cured with wide surgical resection, up to a third of patients will develop metachronous distant metastases [2]. Patients with metastatic STS have limited systemic treatment options and in general a poor prognosis, with median survival averaging one year $[3,4]$.

Although chemotherapy is considered the standard treatment for advanced STS [5], metastasectomy represents an emerging practise in this patient population [5]. Evidence in favour of a surgical approach in the metastatic setting comes from several large retrospective case series investigating overall survival (OS) outcomes of patients who had undergone (mostly pulmonary) metastasectomy [6-23]. These studies have shown very encouraging survival outcomes, far beyond the expected average median survival of around 1 year for metastatic STS. Furthermore, cases with long-term remissions or even potential cure after complete surgical excision of STS metastases have been reported in this context [24]. However, as current studies on metastasectomy in STS are not only retrospective in their nature but also uncontrolled for biases, the question whether metastasectomy improves outcomes as compared to a purely "non-invasive" approach with chemotherapy and supportive measures is currently unknown [25]. Indeed, most evidence on metastasectomy comes from single-centre cohorts within highly specialised tertiary centres involving patients that were likely selected for potentially favourable prognostic factors such as a good performance status, a long metachronous interval, resectable lesions, and/or low metastatic tumour load. Thus, whether favourable outcomes in these cohorts were due to metastasectomy or rather due to the selection of patients with a favourable prognosis for metastasectomy is unclear [25].

While a randomized controlled trial of metastasectomy versus non-invasive treatment would be optimal for quantifying the benefit of surgery in this setting, data from such a trial are unavailable at present, and unlikely to become available in the future due to ethical and logistical reasons [26,27]. In the absence of randomized data, comparative effectiveness studies of observational data may provide guidance for surgeons, medical oncologists and patients [28]. In this study, we perform a comparative effectiveness analysis of surgery versus "non-surgery in patients with metachronous metastases of STS using propensity-score analysis and Inverse Probability of Treatment Weight (IPTW)-modelling. However, even this controlled study is subject to potential selection bias, because treatment assignment to surgery is non-random. A naïve analysis of such data may lead to an overestimation of the potential benefit of metastasectomy. To overcome this limitation, we use advanced comparative effectiveness methods involving propensity scores [29,30].

\section{Patients and methods}

\section{Patients}

In this bi-centre historical cohort study, we retrospectively included 135 patients who were diagnosed with metachronous metastases from histologically-confirmed extremity and trunk STS at two European tertiary centres (Medical University of Graz: $\mathrm{n}=87$, Leiden University Medical Centre: $\mathrm{n}=48$ ). All 135 patients had previously undergone surgery with curative intent for localised STS at these centres, and were drawn from the greater population of 517 patients who had undergone surgery for localised STS between 1998 and 2017 at the Medical University of Graz and 1030 corresponding patients treated between 2000 and 2015 at the Leiden University Medical Centre. Patients with a short follow-up or missing essential information were excluded (Supplementary Figure 1). Two patients initially diagnosed with grade 1 STS were likewise included, as they were subsequently upgraded (Table 1).

Demographic variables and tumour- and treatment-related factors - with special focus on management of metastatic disease - were documented retrospectively as previously described [31,32]. Eastern Cooperative Oncology Group (ECOG) status, as well as haemoglobin and albumin levels, were determined for each patient, as documented at the latest Tumour Board Meeting (TBM) prior to treatment of the metastases.

Date of diagnosis of disseminated disease was defined as the first clinical appointment where imaging studies (i.e. computed tomography, magnetic resonance imaging, chest X-ray, positronemission tomography) provided an indication for metastatic spread that was consecutively confirmed by further metastatic spread, progressive disease or histological examination of resected metastatic nodules. Two study groups were defined retrospectively based on their treatment post diagnosis of metastases: (1) Patients who had undergone any surgical intervention, and (2) patients who had not undergone any surgical intervention. Both groups included patients that received non-invasive treatment approaches, such as chemotherapy or palliative radiotherapy. Treatments were indicated by the multi-disciplinary TBM. Follow-up was calculated from the date of diagnosis of disseminated disease ("baseline date") until death or end of records. Primary endpoint of this study was OS.

\section{Statistical methods}

All statistical analyses were performed using Stata (Windows version 14.0, Stata Corp., Houston, TX, USA). Standardized mean differences (SMDs) were used to quantify differences in means and proportions of variables between the two study groups (surgical intervention vs. no surgical intervention), with SMDs $\geq$ 0.3 being considered indicative of a relevant between-group imbalance. Median follow-up was computed according to the method of Schemper and Smith [29], and OS with a Kaplan-Meier estimator. For comparison of survivor functions between the two study groups, we used log-rank tests. To investigate the association of risk factors with survival, uni- and multivariable Cox models were estimated. The proportionality of hazard assumption was evaluated by fitting an interaction between a variable of interest and linear follow-up time. The propensity score was defined as the probability of undergoing surgical intervention conditional on baseline covariates [30]. This propensity score was predicted from a multivariable logistic regression model (all included covariates are reported in Supplementary Table 1 and are based on clinical experience as well as literature $[4,33,34]$ ). For this propensity score model, missing baseline covariates were imputed with a chained equations algorithm with 10 imputation datasets (list with conditional imputation models available from FP upon request). The inverse-probability-of-treatment-weight (IPTW) was then defined as the inverse of the probability of receiving the treatment that the patient received (also known as the "average treatment effect on the treated"). Following best practise recommendations, SMDs were recalculated after weighing of the data with the IPTWs as a method of balance diagnostics [30]. Here, we pre-specified that the IPTW achieved sufficient balance if an unadjusted SMD $\geq 0.3$ was lowered below this threshold. The primary endpoint of the analysis, i.e. the association between treatment assignment and OS, was then studied with a univariable Cox model which was weighted for the IPTW, as well as IPTW-weighted Kaplan-Meier estimators and log-rank 
Table 1

Baseline characteristics of the study population. Distribution overall and by treatment group.

\begin{tabular}{|c|c|c|c|c|c|c|c|}
\hline & $\mathrm{n}(\%$ missing $)$ & Overall $(\mathrm{n}=135)$ & No surgical intervention $(n=67)$ & Surgical intervention $(n=68)$ & p-value & SMD & SMD-IPTW \\
\hline \multicolumn{8}{|l|}{ Gender } \\
\hline Male & $135(0 \%)$ & 80 (59\%) & $43(64 \%)$ & $37(54 \%)$ & 0.248 & 0.20 & 0.09 \\
\hline Female & & 55 (41\%) & $24(36 \%)$ & $31(46)$ & & & \\
\hline \multicolumn{8}{|l|}{ Location of Primary Tumour } \\
\hline Upper limb & $135(0 \%)$ & 37 (27\%) & $21(31 \%)$ & $16(24 \%)$ & 0.510 & 0.17 & 0.01 \\
\hline Lower limb & & 90 (67\%) & $43(64 \%)$ & 47 (69\%) & & 0.10 & 0.03 \\
\hline Trunk & & $8(6 \%)$ & $3(4 \%)$ & $5(7 \%)$ & & $\mathrm{n} / \mathrm{a}$ & $\mathrm{n} / \mathrm{a}$ \\
\hline \multicolumn{8}{|l|}{ Histology of Primary Tumour } \\
\hline Angiosarcoma & $135(0 \%)$ & $6(4 \%)$ & $4(6 \%)$ & $2(3 \%)$ & 0.557 & $\mathrm{n} / \mathrm{a}$ & $\mathrm{n} / \mathrm{a}$ \\
\hline MPNST & & $13(10 \%)$ & $8(12 \%)$ & $5(7 \%)$ & & $\mathrm{n} / \mathrm{a}$ & $\mathrm{n} / \mathrm{a}$ \\
\hline Myxofibrosarcoma & & $38(28 \%)$ & $18(27 \%)$ & $20(29 \%)$ & & 0.06 & 0.05 \\
\hline Synovial Sarcoma & & $18(13 \%)$ & $9(13 \%)$ & $9(13 \%)$ & & 0.01 & 0.08 \\
\hline UPS & & $10(7 \%)$ & $3(4 \%)$ & $7(10 \%)$ & & $\mathrm{n} / \mathrm{a}$ & $\mathrm{n} / \mathrm{a}$ \\
\hline Spindle cell sarcoma & & $5(4 \%)$ & $1(1 \%)$ & $4(6 \%)$ & & $\mathrm{n} / \mathrm{a}$ & $\mathrm{n} / \mathrm{a}$ \\
\hline Liposarcoma & & $6(4 \%)$ & $2(3 \%)$ & $4(6 \%)$ & & $\mathrm{n} / \mathrm{a}$ & $\mathrm{n} / \mathrm{a}$ \\
\hline Other & & $39(29 \%)$ & $22(33 \%)$ & $17(25 \%)$ & & 0.17 & 0.06 \\
\hline Size of Primary Tumour & $133(2 \%)$ & $10.0[6.0-13.3]$ & $10.0[6.0-13.2]$ & $9.5[5.5-14.0]$ & 0.480 & 0.19 & 0.13 \\
\hline \multicolumn{8}{|l|}{ Grade of Primary Tumour } \\
\hline G1 & $132(2.2 \%)$ & $2(2 \%)$ & $0(0 \%)$ & $2(3 \%)$ & 0.407 & $\mathrm{n} / \mathrm{a}$ & $\mathrm{n} / \mathrm{a}$ \\
\hline G2 & & $23(17 \%)$ & $13(19 \%)$ & $10(15 \%)$ & & 0.11 & 0.04 \\
\hline G3 & & $107(81 \%)$ & $54(81 \%)$ & $53(82 \%)$ & & 0.02 & 0.00 \\
\hline \multicolumn{8}{|l|}{ Surgery Primary Tumour } \\
\hline Limb Salvage & $105(22 \%)$ & 90 (86\%) & 41 (79\%) & 49 (92\%) & 0.055 & 0.39 & 0.04 \\
\hline Amputation & & $15(14 \%)$ & $11(21 \%)$ & $4(8 \%)$ & & & \\
\hline \multicolumn{8}{|l|}{ Adjuvant Therapy } \\
\hline No Adjuvant Therapy & $96(29 \%)$ & $25(26 \%)$ & $16(33 \%)$ & $9(19 \%)$ & 0.356 & 0.31 & 0.06 \\
\hline (Neo-)Adjuvant RTX & & $61(64 \%)$ & $27(55 \%)$ & $34(72 \%)$ & & 0.36 & 0.08 \\
\hline (Neo-)Adjuvant CTX & & $3(3 \%)$ & $2(4 \%)$ & $1(2 \%)$ & & $\mathrm{n} / \mathrm{a}$ & $\mathrm{n} / \mathrm{a}$ \\
\hline Both & & $7(7 \%)$ & $4(8 \%)$ & $3(6 \%)$ & & $\mathrm{n} / \mathrm{a}$ & $\mathrm{n} / \mathrm{a}$ \\
\hline \multicolumn{8}{|l|}{ Count of Metastases } \\
\hline Singular & $135(0 \%)$ & $51(38 \%)$ & $15(22 \%)$ & $36(53 \%)$ & $<0.0001$ & 0.66 & 0.15 \\
\hline Multiple & & $84(62 \%)$ & $52(48 \%)$ & $32(47 \%)$ & & & \\
\hline Number of Metastases* & $135(0 \%)$ & $2[1-9]$ & $6[2-9]$ & $1[1-3]$ & $<0.0001$ & 0.96 & 0.20 \\
\hline Age at Metastasis (Years) & $135(0 \%)$ & $65[50-75]$ & $67[52-78]$ & $63[47-74]$ & 0.158 & 0.25 & 0.07 \\
\hline Time to 1st Metastasis (Months) & $134(0.7 \%)$ & $11[4-20]$ & $7[3-20]$ & $14[6-25]$ & 0.020 & 0.11 & 0.02 \\
\hline \multicolumn{8}{|l|}{ Location of Metastasis** } \\
\hline Lungs & $134(0.7 \%)$ & $99(74 \%)$ & $56(85 \%)$ & $43(63 \%)$ & 0.039 & 0.50 & 0.15 \\
\hline Soft tissues + LN & & $21(16 \%)$ & $4(6 \%)$ & $17(25 \%)$ & & 0.54 & 0.17 \\
\hline Bone & & $7(5 \%)$ & $3(5 \%)$ & $4(6 \%)$ & & $\mathrm{n} / \mathrm{a}$ & $\mathrm{n} / \mathrm{a}$ \\
\hline Organs & & $2(1 \%)$ & $1(1.5 \%)$ & $1(1 \%)$ & & $\mathrm{n} / \mathrm{a}$ & $\mathrm{n} / \mathrm{a}$ \\
\hline Skip lesion & & $5(4 \%)$ & $2(3 \%)$ & $3(4 \%)$ & & $\mathrm{n} / \mathrm{a}$ & $\mathrm{n} / \mathrm{a}$ \\
\hline Haemoglobin (g/dL) & $98(27 \%)$ & $12.9[11.0-14.3]$ & $12.6[10.6-13.3]$ & $13.5[11.5-14.8]$ & 0.006 & 0.57 & 0.22 \\
\hline Albumin $(\mathrm{g} / \mathrm{dL})$ & $61(55 \%)$ & $4.2[3.5-4.6]$ & $4.0[3.2-4.2]$ & $4.5[4.0-4.8]$ & 0.002 & 0.81 & 0.35 \\
\hline ECOG PS & $103(24 \%)$ & $1[0-1]$ & $1[0-2]$ & $0.5[0-1]$ & 0.019 & 0.54 & 0.27 \\
\hline
\end{tabular}

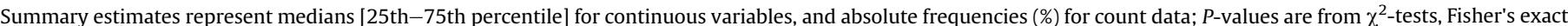

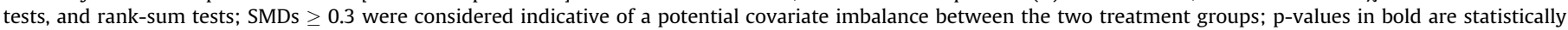

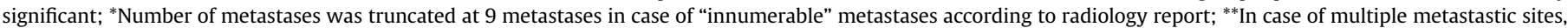

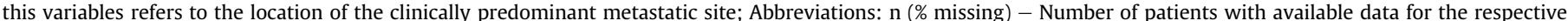

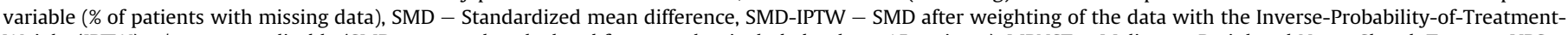

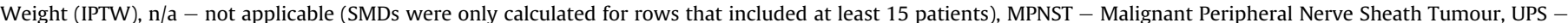

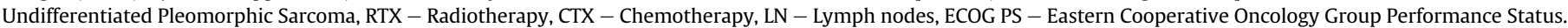

tests [35]. Importantly, metastasectomy was treated as a timedependent variable in this main analysis to account for potential immortal time bias due to the time between metastasis diagnosis and metastasectomy [36-38]. Key prognostic variables and variables which did not achieve an SMD $<0.3$ after IPTWweighting were subsequently included in this Cox model to account for potential residual confounding. In a sensitivity analysis, we used a "truncated" IPTW excluding patients with an IPTW $<5 \%$ or $>95 \%$ of the IPTW's distribution [30]. To study potential predictive biomarkers for benefit from surgical intervention, we fitted interactions between treatment assignment and several clinical and laboratory markers. Due to the low power of interaction tests and the moderately large sample size, a $\mathrm{p}<0.1$ was considered to indicate statistical significance for this interaction analysis. To further control for potential immortal time bias due to the time between metastasis diagnosis and metastasectomy, we finally performed landmark analyses with the landmark set at
3 and 6 months after diagnosis of metastases [36-39]. The full analysis code is available on request from FP.

\section{Results}

\section{Baseline characteristics}

The median age of the cohort at diagnosis of metachronous metastases was 65 years [25th-75th percentile: 50-75], and 55 patients $(41 \%)$ were female. Further patient characteristics are found in Table 1 .

On average, patients developed metastatic disease after a median follow-up of 11 months (IQR: 4-20 months). Ninety-nine patients first presented with lung metastases (73\%), 21 with metastases to soft tissues and lymph nodes (16\%) and 7 patients with metastases to bone (5\%). Furthermore, 5 patients had skip lesions (4\%) and 2 patients presented with intestinal metastases (1\%). 
Information on location of metastases was missing in one patient (1\%). Fifty-one patients had one metastatic lesion only (38\%) while 84 patients presented with multiple metastases (62\%). Fifty-nine patients developed further metastases after diagnosis of the first metastasis (44\%).

Of the 99 patients with primary metastases to the lungs, 28 presented with a singular pulmonary nodule (28\%) and 71 with multiple lesions (72\%). Forty-seven patients had pulmonary nodules restricted to one side (48\%) and 51 patients had metastatic lesions in both lungs (52\%). In one patient, the involvement of the other side was uncertain.

Altogether, 67 patients (50\%) were not treated surgically, of whom 32 received best supportive care (BSC) and 35 were administered CTX \pm RTX. The remaining 68 patients underwent surgery (50\%, Supplementary Table 2 ). Surgical interventions in the metastatic setting included wedge-resections $(n=16,23 \%)$ and lobectomies ( $\mathrm{n}=28,41 \%$ ) of lung metastases, as well as (lymph node) extirpations ( $n=10,15 \%)$ and resections $(n=14,21 \%)$ for peripheral metastases.

As expected, patients in the surgical group had a significantly higher baseline prevalence of favourable prognostic factors as compared to patients in the non-surgery group. For example, patients who underwent surgery had less metastatic lesions (median: 1 vs. 6 [SMD $=0.96]$ ), a better ECOG performance status (median: 0.5 vs. 1 [SMD $=0.54]$ ), and higher haemoglobin levels (median: 13.5 vs. $12.6 \mathrm{~g} / \mathrm{dL}$ [SMD = 0.57], Table 1).

After the diagnosis of metachronous metastases, patients were followed up for a median interval of 4.9 years (range: 1 day-16.6 years). Seventy-five percent and $25 \%$ of the cohort were followed up for at least 1.9 years and 9.3 years, respectively. During followup we observed 89 deaths (66\%), corresponding to 1-, 5-, and 10year OS estimates of $63 \%, 24 \%$, and 15\% (Supplementary Figure 2). Seventy-three (82\%) of these 89 deaths were due to disease progression, five deaths (6\%) were due to other causes, while cause of death was unknown in 11 cases (12\%). Prognostic factors for OS in the univariate setting are reported in Table 2. The strongest univariable predictors of worse OS were higher ECOG performance status, upper limb tumour location, a higher number of metastases, the presence of brain metastases, anaemia, and hypoalbuminaemia, respectively.

\section{Surgery and OS in metachronous metastases of STS}

In the unadjusted analysis, OS was significantly longer in those 68 patients who had undergone surgical interventions compared to the 67 patients who had not (Fig. 1). One-, 5-, and 10-year OSestimates were $83 \%, 34 \%$ and $23 \%$ in the surgery group, and $38 \%$, $11 \%$ and $4 \%$ in the non-surgery group, respectively (log-rank$\mathrm{p}<0.0001)$. Median OS was 2.7 years since diagnosis of first metastasis (95\% CI: 1.6-3.9) in the surgery group, and 0.8 years (95\% CI: $0.4-1.2$ ) in the non-surgery group, respectively.

To account for the significantly higher prevalence of favourable prognostic factors in the surgery group (consistent with a nonrandom assignment to the two treatment groups), we predicted a propensity score and an inverse-probability-of-treatment-weight (IPTW, see Supplementary Table 1 and Supplementary Fig. 3A, B). Re-weighting of the data removed nearly all imbalances in key prognostic variables between the two groups, except for albumin (Table 1). For example, the SMD for the number of metastatic lesions was reduced from 0.96 to 0.20 , for ECOG performance status from 0.54 to 0.27 , and for haemoglobin from 0.57 to 0.22 , respectively.

The favourable association between any surgical intervention and lower risk of death prevailed after re-weighting the data for the IPTW. In detail, the IPTW-weighted 1-, 5-, and 10-year OSestimates were $86 \%, 31 \%$ and $17 \%$ in the surgery group, and $39 \%$, $10 \%$ and $3 \%$ in the non-surgery group, respectively (log-rank$\mathrm{p}<0.0001$, Fig. 2). The corresponding IPTW-adjusted median OS estimates were 3.3 years and 0.9 years in the surgery and nonsurgery group, respectively. Further multivariable adjustment for other relevant predictors of worse OS, such as poor ECOG performance status, higher number of metastases, low haemoglobin and low albumin did not alter this association (adjusted HR for metastasectomy treated as a time-dependent variable $=0.55,0.30-0.98$, Table 3 ).

\section{Exploration of potential predictive factors for benefit from metastasectomy}

We fitted interactions between treatment assignment and selected clinical covariates within IPTW-adjusted Cox-models to

Table 2

Univariable Analysis of Prognostic Factors for Overall Survival (OS).

\begin{tabular}{|c|c|c|c|}
\hline Variable & HR & $95 \% \mathrm{CI}$ & p-value \\
\hline Female gender & 0.99 & $0.65-1.52$ & 0.971 \\
\hline Age at metastasis (per 5 years increase) & 1.03 & $0.97-1.09$ & 0.414 \\
\hline ECOG performance status (per 1 point increase) & 1.81 & $1.29-2.56$ & 0.001 \\
\hline \multicolumn{4}{|l|}{ Primary tumour location } \\
\hline Upper limb & Ref. & Ref. & 0.016 \\
\hline Lower limb & 0.55 & $0.35-0.87$ & \\
\hline Trunk & 1.25 & $0.52-3.01$ & \\
\hline Tumour grade G3 & 1.49 & $0.86-2.57$ & 0.155 \\
\hline Multiple metastases & 1.47 & $0.95-2.27$ & 0.081 \\
\hline Number of metastases* (per 1 metastasis increase) & 1.07 & $1.00-1.13$ & 0.039 \\
\hline Time to 1 st metastasis (per 5 months increase $e^{* *}$ ) & 0.98 & $0.93-1.04$ & 0.546 \\
\hline \multicolumn{4}{|l|}{ Metastasis location } \\
\hline Any lung metastasis & 2.05 & $1.19-3.55$ & 0.010 \\
\hline Any soft tissue and/or lymph node metastasis & 0.62 & $0.36-1.06$ & 0.079 \\
\hline Any bone metastasis & 1.35 & $0.72-2.55$ & 0.354 \\
\hline Any solid organ metastasis (excluding lung and brain) & 1.32 & $0.70-2.49$ & 0.388 \\
\hline Any brain metastasis & 1.97 & $1.07-3.63$ & 0.030 \\
\hline Limb amputation upon primary surgery & 1.68 & $0.88-3.24$ & 0.118 \\
\hline Haemoglobin (per $1 \mathrm{~g} / \mathrm{dL}$ increase) & 0.87 & $0.78-0.98$ & 0.019 \\
\hline Albumin (per $1 \mathrm{~g} / \mathrm{dL}$ increase) & 0.56 & $0.35-0.89$ & 0.016 \\
\hline
\end{tabular}

Estimates were obtained with univariable Cox proportional hazards models in multiply-imputed data. p-values in bold are statistically significant; *Number of metastases was truncated at 9 metastases in case of "innumerable" metastases according to radiology report; ** The date of primary surgery was used as the baseline date for calculation of the "time to 1st metastasis" variable. Abbreviations: HR - Hazard ratio, 95\% CI - 95\% confidence interval, p - Wald-test p-value, ECOG - Eastern Cooperative Oncology Group. 


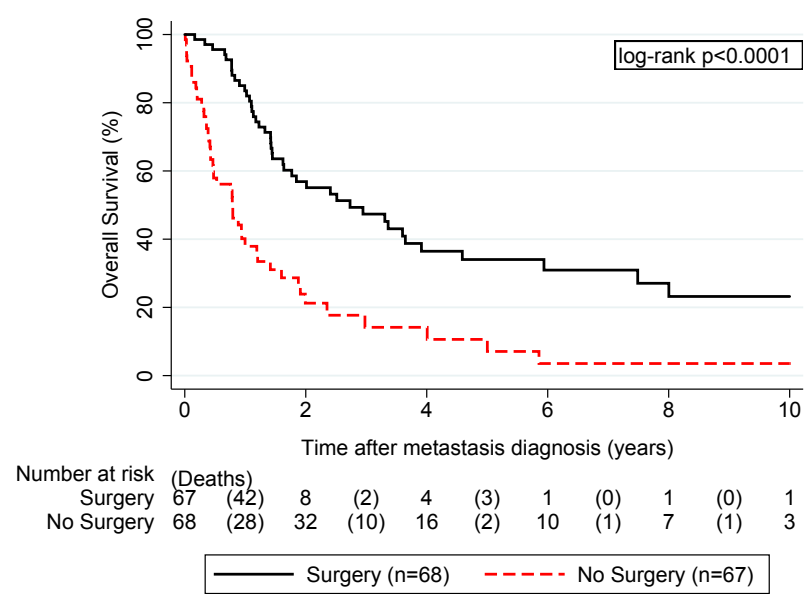

Fig. 1. Crude Kaplan-Meier Overall Survival (OS) functions by treatment group for metastasis. Numbers in round brackets in the risk table report the number of deaths in the respective time interval.

identify potential subgroups with a very high or low benefit from surgical intervention (Table 4). In this analysis of predictive markers, the potential benefit of surgical intervention was consistent across important clinical subgroups such as patients (1) with and without poor ECOG performance status, (2) a metachronous interval of $\geq$ or $<12$ months or (3) an age $\geq$ or $<70$ years at metastasis diagnosis. Importantly, with the modified threshold of statistical significance of $p<0.1$ for this interaction analysis, the benefit of metastasectomy was stronger in patients with a single metastasis $(\mathrm{HR}=0.21)$ than in patients with multiple metastases $(\mathrm{HR}=0.44$, $\mathrm{p}$ for interaction $=0.096$ ). Although not statistically significant, the regression coefficients at least pointed in the direction of metastasectomy being slightly more efficacious in patients with a metachronous interval of at least 12 months.

\section{Landmark analysis}

The median time between metastasis diagnosis and metastasectomy was 1.7 months [IQR: $0.7-3.6]$. In previous analyses, this potential immortal time bias was controlled for by treating metastasectomy as a time-dependent covariable. In a sensitivity

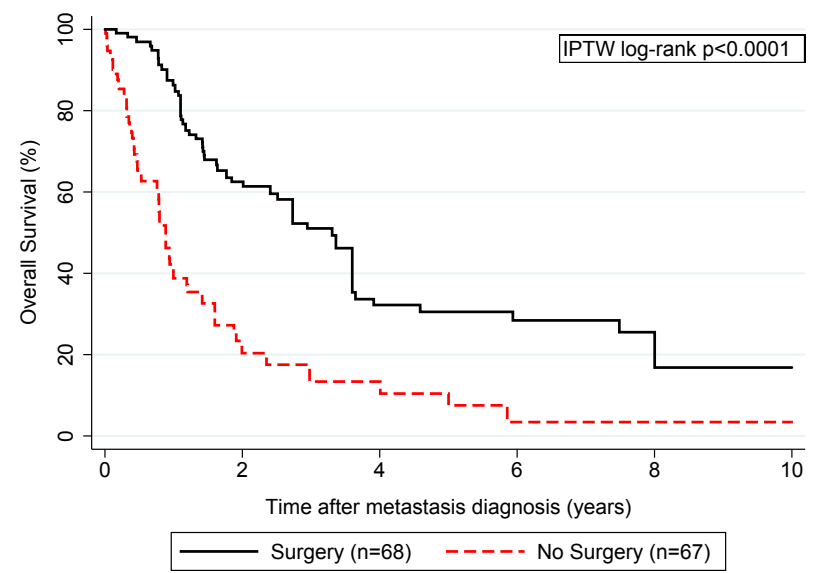

Fig. 2. Inverse-probability-of-treatment-weighted (IPTW) Kaplan-Meier Overall Survival (OS) functions by treatment group. No risk table is displayed because the numbers of patients at specific time points are non-integers in an IPTW-weighted sample.
Table 3

Multivariable IPTW-weighted analysis of Overall Survival (OS).

\begin{tabular}{llll}
\hline Variable & HR & $95 \% \mathrm{CI}$ & p-value \\
\hline Multivariable Cox regression Model & & & \\
Metastasectomy as a time-dependent covariate & 0.55 & $0.30-0.98$ & $\mathbf{0 . 0 4 3}$ \\
ECOG performance status (per 1 point increase) & 1.72 & $1.22-2.42$ & $\mathbf{0 . 0 0 2}$ \\
Number of metastases* (per 1 metastasis increase) & 0.99 & $0.91-1.07$ & 0.730 \\
Haemoglobin (per 1 g/dL increase) & 1.00 & $0.83-1.20$ & 0.984 \\
Albumin (per 1 g/dL increase) & 0.81 & $0.39-1.65$ & 0.541 \\
\hline
\end{tabular}

Multivariable Model\#1 is a multivariable Cox model, which was weighted with the Inverse-Probability-of-Treatment-Weight (IPTW). Multivariable Model \#2 is also an IPTW-weighted multivariable Cox Model. with, metastasectomy was treated as a time-dependent covariate Both models were estimated from multiply-imputed data. p-values in bold are statistically significant: *Number of metastases was truncated at 9 metastases in case of "innumerable" metastases according to radiology report. Abbreviations: $\mathrm{HR}$ - Hazard ratio, 95\% CI - 95\% confidence interval, $\mathrm{p}$ - Wald-test p-value, ECOG - Eastern Cooperative Oncology Group.

analysis of immortal time bias, we performed IPTW--weighted landmark analyses with landmarks at 3 and 6 months after metastasis diagnosis, respectively. With a landmark at 3 months, the 5 -year OS estimates were $27.8 \%$ and $14.5 \%$ in the surgery and non-surgery group, respectively (Fig. 3A), and similar results were observed for a landmark at 6 months (Fig. 3B).

\section{Discussion}

Previous non-controlled observational studies have reported very favourable survival outcomes in patients with metastatic STS who underwent metastasectomy [6-23]. However, whether these encouraging results are attributable to metastasectomy or simply due to selection bias has so far not been adequately proven [25-27]. Aiming to fill this gap of evidence, we performed a comparative effectiveness analysis of surgery versus non-surgery for patients with metachronous metastasis of STS. Using propensity score methods, landmark analysis, and timedependent Cox modelling to control for selection and immortal time bias, we observed that metastasectomy was associated with prolonged overall survival. In the absence of randomisation, these data support the hypothesis that surgery may improve survival of patients with metachronous metastases of STS.

An important aspect of STS-management is the therapeutic approach in the metastatic setting. Depending on the patients' general condition, site of metastases and overall tumour load, nonsurgical interventions (i.e. BSC, CTX, RTX) or a surgical approach may be chosen $[40,41]$. In our cohort, half of the patients underwent a surgical intervention for their metastases, about one quarter received $\mathrm{RTX} \pm \mathrm{CTX}$ and another quarter received BSC. Consistent with the literature, the most common location of primary metastasis in our cohort was the lung, followed by soft tissues and lymph nodes $[6,34,42,43]$.

In the univariate setting, patients undergoing surgical interventions had significantly better 5- and 10-year survival of 34\% and $23 \%$ vs. $11 \%$ and $4 \%$ for the non-surgery group (BSC $\pm \mathrm{RTX} \pm \mathrm{CTX}$ ), respectively. These data are in line with the observations made by Billingsley et al. in a large retrospective cohort of STS-patients with pulmonary metastases [6].

Given the retrospective design of this study and the noncontrolled design of many studies performed in the past $[6-23,33,34,44,45]$, one may argue that the benefit of surgical intervention can be explained by favourable clinical parameters prevailing in the surgical-group. Indeed, patients undergoing surgical resection in our cohort presented with fewer metastases and a longer interval between initial surgery and occurrence of metastasis, and also had a better ECOG performance status and higher haemoglobin level at the time of treatment decision. These features 
Table 4

Predictive factors for overall survival (OS) benefit from metastasectomy - Interaction analysis.

\begin{tabular}{|c|c|c|c|}
\hline & Hazard ratio & $95 \% \mathrm{CI}$ & Interaction p-value \\
\hline ECOG performance status: 0 points & 0.43 & $0.18-1.02$ & 0.373 \\
\hline ECOG performance status: $1-2$ points & 0.27 & $0.13-0.42$ & \\
\hline Time between primary surgery and metastasis onset $<12$ months & 0.49 & $0.21-0.78$ & 0.154 \\
\hline Time between primary surgery and metastasis onset $\geq 12$ months & 0.27 & $0.14-0.50$ & \\
\hline Age $<70$ years & 0.36 & $0.21-0.62$ & 0.678 \\
\hline Age $\geq 70$ years & 0.30 & $0.15-0.59$ & \\
\hline Number of metastases: Single & 0.21 & $0.10-0.43$ & 0.096 \\
\hline Number of metastases: Multiple & 0.44 & $0.26-0.77$ & \\
\hline
\end{tabular}

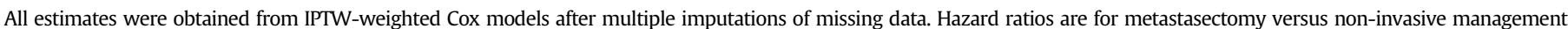

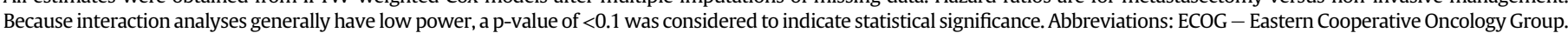
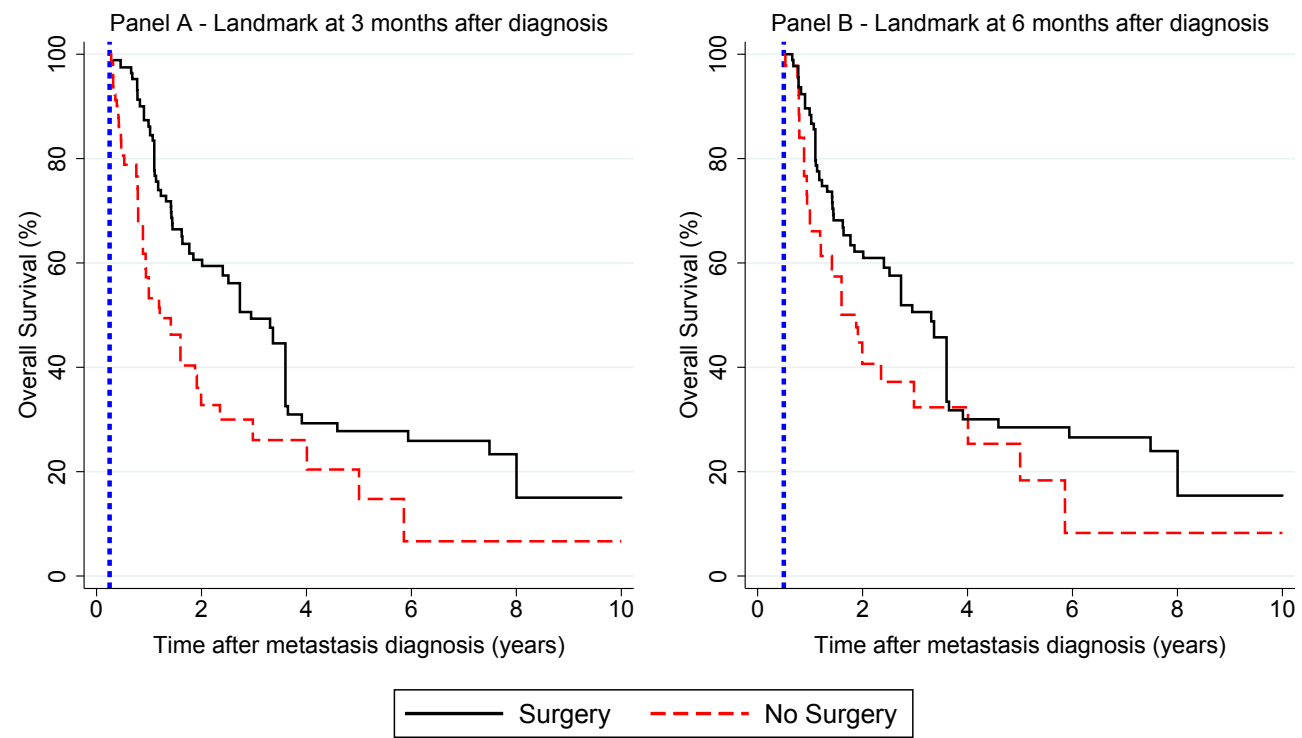

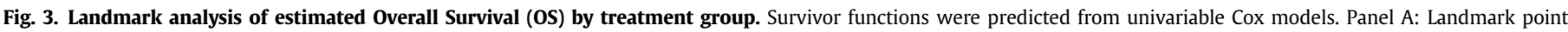
(short-dashed blue vertical line) set at 3 months after metastasis diagnosis. Panel B: Landmark point set at 6 months after metastasis diagnosis.

have already been identified as prognostic factors for prolonged survival of STS-patients with metastatic disease [4,33,34].

Therefore, we used a propensity score approach with IPTWweighting to compensate for differences at treatment decision between patients undergoing surgical interventions and those who did not. After weighting all patients for the IPTW-score and recalculating the uni- and multivariable time-to-death analyses, any surgical intervention remained a significant positive prognostic factor for post-metastasis-survival independent of ECOG performance status, number of metastases, haemoglobin- and albuminlevels. The magnitude of association of surgery with OS was large, suggesting that patients with metachronous metastases from STS may gain more than two years in median OS from metastasectomy. The potential benefit of surgery even prevailed in the landmark and time-dependent Cox regression analyses in which immortal time bias due to time between metastasis diagnosis and surgery was accounted for.

By fitting interactions between clinically relevant baseline covariates and treatment assignment, we aimed to identify predictive markers of benefit from surgery. This analysis suggested that the benefit of surgery was greater for patients with singular metastasis at the time of surgery, but was otherwise consistent across several clinically-meaningful subgroups.

Our study is not without limitations. Due to its retrospective design, the study depends on the quality and availability of medical records, pathology reports and medical images. Moreover, the validity of the IPTW analysis depends on the difficult-to-test assumption that the propensity score model is correctly specified [35]. Furthermore, other hidden confounding factors, such as comorbidities not covered by the ECOG performance status, could not be considered in the calculations. Otherwise, a major strength of our analysis is that all study patients were drawn from established cohorts at Graz and Leiden that included all consecutive patients who had previously undergone resection with curative intent for localised STS. With this approach, we could reduce selection bias in both surgery and non-surgery groups.

\section{Conclusion}

This comparative effectiveness analysis of observational data using propensity score methods supports the hypothesis that surgery may be an efficacious treatment option for metachronous metastases of STS with a potentially sizeable benefit in terms of improving overall survival. Moreover, the potential benefit of surgical intervention appears to be consistent across clinically important subgroups, although surgery may be less efficacious in patients with multiple metastases. These data should be taken into account by clinicians treating sarcomas and their patients when planning treatment for metachronous metastases of STS.

\section{Conflicts of interest statement}

Florian Posch has received support in kind for any aspect by MSD Oncology, GWT TUD GmbH, PharmaMar, Novartis, Ipsen, 
Pfizer, OctaPharma and Astellas. Michiel van de Sande received grants for his institution from the National Cancer Fund and Daichi Sankyo. Veroniek van Praag has received grants for her institution from the Dutch Cancer Society (DCS). Andreas Leithner has received grants from Johnson\&Johnson, Medtronic, Alphamed and Zimmer. The remaining authors have no conflicts of interest to declare.

\section{Appendix A. Supplementary data}

Supplementary data related to this article can be found at https://doi.org/10.1016/j.ejso.2018.06.019.

\section{References}

[1] Fletcher CD. Pathology of soft tissue sarcomas. Edinburgh, Scotland: Churchill Livingstone; 1990.

[2] Posch F, Leitner L, Bergovec M, Bezan A, Stotz M, Gerger A, et al. Can multistate modeling of local recurrence, distant metastasis, and death improve the prediction of outcome in patients with soft tissue sarcomas? Clin Orthop Relat Res 2017;475:1427-35.

[3] Van Glabbeke M, van Oosterom AT, Oosterhuis JW, Mouridsen H, Crowther D, Somers R, et al. Prognostic factors for the outcome of chemotherapy in advanced soft tissue sarcoma: an analysis of 2,185 patients treated with anthracycline-containing first-line regimens-a European Organization for Research and Treatment of Cancer Soft Tissue and Bone Sarcoma Group Study. J Clin Oncol 1999:17:150-7.

[4] Italiano A, Mathoulin-Pelissier S, Cesne AL, Terrier P, Bonvalot S, Collin F, et al Trends in survival for patients with metastatic soft-tissue sarcoma. Cancer 2011;117:1049-54.

[5] Group ESESNW. Soft tissue and visceral sarcomas: ESMO Clinical Practice Guidelines for diagnosis, treatment and follow-up. Ann Oncol 2014;25(Suppl. 3):iii102-12.

[6] Billingsley KG, Burt ME, Jara E, Ginsberg RJ, Woodruff JM, Leung DH, et al. Pulmonary metastases from soft tissue sarcoma: analysis of patterns of diseases and postmetastasis survival. Ann Surg 1999;229:602-10. Discussion 10-12.

[7] Mizuno T, Taniguchi T, Ishikawa Y, Kwaguchi K, Fukui T, Ishiguro F, et al. Pulmonary metastasectomy for osteogenic and soft tissue sarcoma: who really benefits from surgical treatment? Eur J Cardio Thorac Surg 2013;43:795-9.

[8] Okiror L, Peleki A, Moffat D, Bille A, Bishay E, Rajesh P, et al. Survival following pulmonary metastasectomy for sarcoma. Thorac Cardiovasc Surg 2016;64:146-9.

[9] Schur S, Hoetzenecker K, Lamm W, Koestler WJ, Lang G, Amann V, et al. Pulmonary metastasectomy for soft tissue sarcoma-report from a dual institution experience at the Medical University of Vienna. Eur J Cancer 2014;50:2289-97.

[10] Dossett LA, Toloza EM, Fontaine J, Robinson LA, Reed D, Druta M, et al. Outcomes and clinical predictors of improved survival in a patients undergoing pulmonary metastasectomy for sarcoma. J Surg Oncol 2015;112:103-6.

[11] Kim S, Ott HC, Wright CD, Wain JC, Morse C, Gaissert HA, et al. Pulmonary resection of metastatic sarcoma: prognostic factors associated with improved outcomes. Ann Thorac Surg 2011:92:1780-6. Discussion 6-7.

[12] Toussi MS, Bagheri R, Dayani M, Anvari K, Sheibani S. Pulmonary metastasectomy and repeat metastasectomy for soft-tissue sarcoma. Asian Cardiovasc Thorac Ann 2013;21:437-42.

[13] Predina JD, Puc MM, Bergey MR, Sonnad S, Kucharczuk JC, Staddon A, et at. Improved survival after pulmonary metastasectomy for soft tissue sarcoma. J Thorac Oncol 2011;6:913-9.

[14] Giuliano K, Sachs T, Montgomery E, Guzzetta A, Brock M, Pawlik TM, et al. Survival following lung metastasectomy in soft tissue sarcomas. Thorac Cardiovasc Surg 2016;64:150-8.

[15] Rehders A, Hosch SB, Scheunemann P, Stoecklein NH, Knoefel WT, Peiper M. Benefit of surgical treatment of lung metastasis in soft tissue sarcoma. Arch Surg 2007;142:70-5. Discussion 6.

[16] van Geel AN, Pastorino U, Jauch KW, Judson IR, van Coevorden F, Buesa JM, et al. Surgical treatment of lung metastases: the European organization for research and treatment of cancer-soft tissue and bone sarcoma group study of 255 patients. Cancer 1996;77:675-82.

[17] Ueda T, Uchida A, Kodama K, Doi O, Nakahara K, Fujii Y, et al. Aggressive pulmonary metastasectomy for soft tissue sarcomas. Cancer 1993;72: $1919-25$.

[18] Choong PF, Pritchard DJ, Rock MG, Sim FH, Frassica FJ. Survival after pulmonary metastasectomy in soft tissue sarcoma. Prognostic factors in 214 patients. Acta Orthop Scand 1995;66:561-8.

[19] Garcia Franco CE, Algarra SM, Ezcurra AT, Guillen-Grima F, San-Julian M, Mindan JP, et al. Long-term results after resection for soft tissue sarcoma pulmonary metastases. Interact Cardiovasc Thorac Surg 2009;9:223-6.
[20] Marudanayagam R, Sandhu B, Perera MT, Bramhall SR, Mayer D, Buckels JA, et al. Liver resection for metastatic soft tissue sarcoma: an analysis of prognostic factors. Eur J Surg Oncol 2011;37:87-92.

[21] Pawlik TM, Vauthey JN, Abdalla EK, Pollock RE, Ellis LM, Curely SA. Results of a single-center experience with resection and ablation for sarcoma metastatic to the liver. Arch Surg 2006;141:537-43. Discussion 43-44.

[22] Nakamura T, Matsumine A, Yamakado K, Takao M, Uchida A, Sudo A. Clinical significance of radiofrequency ablation and metastasectomy in elderly patients with lung metastases from musculoskeletal sarcomas. J Cancer Res Ther 2013;9:219-23.

[23] Falk AT, Moureau-Zabotto L, Oali M, Penel N, Italiano A, Bay JO, et al. Effect on survival of local ablative treatment of metastases from sarcomas: a study of the French sarcoma group. Clin Oncol (R Coll Radiol) 2015;27:48-55.

[24] Digesu CS, Wiesel O, Vaporciyan AA, Colson YL. Management of sarcoma metastases to the lung. Surg Oncol Clin N Am 2016;25:721-33.

[25] Berry MF. Evidence for resection of sarcoma pulmonary metastases: more, but better? J Thorac Cardiovasc Surg 2017;154:317-8.

[26] Chudgar NP, Brennan MF, Munhoz RR, Bucciarelli PR, Tan KS, D'Angelo SP, et al. Pulmonary metastasectomy with therapeutic intent for soft-tissue sarcoma. J Thorac Cardiovasc Surg 2017;154:319-330 e1.

[27] Hoang CD. Resection of pulmonary sarcomatous metastases: cut to cure? J Thorac Cardiovasc Surg 2017;154:331.

[28] Visvanathan K, Levit LA, Raghavan D, Hudis CA, Wong S, Dueck A, et al. Untapped potential of observational research to inform clinical decision making: American society of clinical Oncology research statement. J Clin Oncol 2017;35:1845-54.

[29] Schemper M, Smith TL. A note on quantifying follow-up in studies of failure time. Contr Clin Trials 1996;17:343-6.

[30] Austin PC, Stuart EA. Moving towards best practice when using inverse probability of treatment weighting (IPTW) using the propensity score to estimate causal treatment effects in observational studies. Stat Med 2015;34: 3661-79.

[31] Smolle MA, Tunn PU, Goldenitsch E, Posch F, Szkandera J, Bergovec M, et al. The prognostic impact of unplanned excisions in a cohort of 728 soft tissue sarcoma patients: a multicentre study. Ann Surg Oncol 2017 Jun;24(6): $1596-605$.

[32] van Praag VM, Rueten-Budde AJ, Jeys LM, Laitinen MK, Pollock R, Aston W, et al. A prediction model for treatment decisions in high-grade extremity softtissue sarcomas: personalised sarcoma care (PERSARC). Eur J Cancer 2017;83: $313-23$.

[33] Iqbal N, Shukla NK, Deo SV, Agarwala S, Sharma DN, Sharma MC, et al. Prognostic factors affecting survival in metastatic soft tissue sarcoma: an analysis of 110 patients. Clin Transl Oncol 2016;18:310-6.

[34] Ferguson PC, Deheshi BM, Chung P, Catton CN, O'Sullivan B, Gupta A, et al. Soft tissue sarcoma presenting with metastatic disease: outcome with primary surgical resection. Cancer 2011;117:372-9.

[35] Austin PC. The use of propensity score methods with survival or time-to-event outcomes: reporting measures of effect similar to those used in randomized experiments. Stat Med 2014;33:1242-58.

[36] Putter H, Fiocco M, Geskus RB. Tutorial in biostatistics: competing risks and multi-state models. Stat Med 2007:26:2389-430.

[37] Dafni U. Landmark analysis at the 25-year landmark point. Circ Cardiovasc Qual Outcomes 2011;4:363-71.

[38] Posch F, Riedl J, Reitter EM, Kaider A, Zielinski C, Pabinger I, et al. Hypercoagulabilty, venous thromboembolism, and death in patients with cancer. A multi-state model. Thromb Haemost 2016;115:817-26.

[39] de Wreede LC, Fiocco M, Putter H. The mstate package for estimation and prediction in non- and semi-parametric multi-state and competing risks models. Comput Meth Progr Biomed 2010;99:261-74.

[40] Blackmon SH, Shan N, Roth JA, Correa AM, Vaprociyan AA, Rice DC, et al. Resection of pulmonary and extrapulmonary sarcomatous metastases is associated with long-term survival. Ann Thorac Surg 2009;88:877-84. Discussion 84-85.

[41] Casali PG, Blay JY. Soft tissue sarcomas: ESMO Clinical Practice Guidelines for diagnosis, treatment and follow-up. Ann Oncol 2010;21(Suppl. 5): v198-203.

[42] Gadd MA, Casper ES, Woodruff JM, McCormack PM, Brennan MF. Development and treatment of pulmonary metastases in adult patients with extremity soft tissue sarcoma. Ann Surg 1993:218:705-12.

[43] Coindre JM, Terrier P, Guillou L, Le Doussal V, Collin F, Ranchere D, et al, Predictive value of grade for metastasis development in the main histologic types of adult soft tissue sarcomas: a study of 1240 patients from the French Federation of Cancer Centers Sarcoma Group. Cancer 2001:91:1914-26.

[44] Biswas B, Shukla NK, Deo SV, Agarwala S, Sharma DN, Vishnubhatla S, et al. Evaluation of outcome and prognostic factors in extraosseous Ewing sarcoma. Pediatr Blood Cancer 2014:61:1925-31.

[45] Smith R, Pak Y, Kraybill W, Kane JM. Factors associated with actual long-term survival following soft tissue sarcoma pulmonary metastasectomy. Eur J Surg Oncol 2009;35:356-61. 